\title{
A DuMond-type crystal spectrometer for synchrotron-based X-ray emission studies in the energy range of 15-26 keV
}

\author{
P. Jagodziński, ${ }^{1,2,3, a)}$ J. Szlachetko, ${ }^{4}$ (D) J.-Cl. Dousse, ${ }^{5}$ J. Hoszowska, ${ }^{5}$ M. Szlachetko, ${ }^{1}$ U. Vogelsang, ${ }^{1}$ D. Banaś, ${ }^{2}$ \\ T. Pakendorf, ${ }^{6}$ A. Meents, ${ }^{6}$ J. A. van Bokhoven, ${ }^{1}$ A. Kubala-Kukuś, ${ }^{2}$ M. Pajek, $^{2}$ and M. Nachtegaal ${ }^{1, b)}$
}

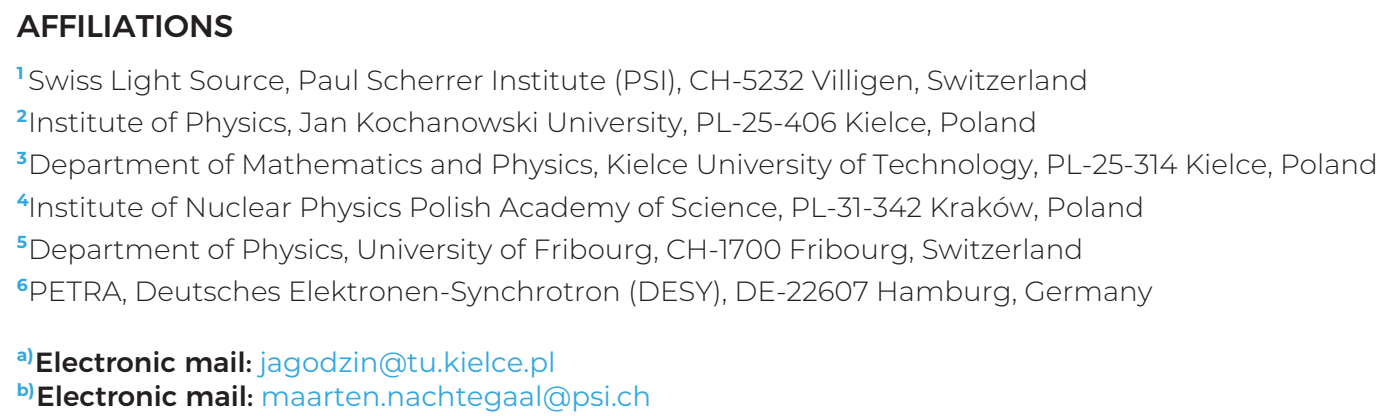

\section{ABSTRACT}

The design and performance of a high-resolution transmission-type X-ray spectrometer for use in the 15-26 keV energy range at synchrotron light sources is reported. Monte Carlo X-ray-tracing simulations were performed to optimize the performance of the transmission-type spectrometer, based on the DuMond geometry, for use at the Super X-ray absorption beamline of the Swiss Light Source at the Paul Scherrer Institute. This spectrometer provides an instrumental energy resolution of $3.5 \mathrm{eV}$ for X-ray emission lines around $16 \mathrm{keV}$ and $12.5 \mathrm{eV}$ for emission lines at $26 \mathrm{keV}$, which is comparable to the natural linewidths of the $K$ and $L \mathrm{X}$-ray transitions in the covered energy range. First experimental data are presented and compared with results of the Monte Carlo X-ray simulations.

\section{INTRODUCTION}

X-ray absorption (XAS) and X-ray emission (XES) spectroscopies represent the most commonly used techniques in X-ray spectroscopy. ${ }^{1}$ They can be employed as element specific tools to probe the local geometric and electronic structures of most elements throughout the periodic table in solid, liquid, and gaseous samples. In XAS measurements, core level electrons are excited into unfilled outer subshells or promoted into the continuum. XAS permits therefore to probe the density of unoccupied electronic states. In XES, core vacancies are filled by outer shell electrons thereby emitting photons with characteristic energies so that XES allows us to probe the density of occupied electronic states. XAS and XES can thus be considered as complementary techniques. ${ }^{2}$
XES is a powerful tool used in many scientific fields including chemistry, biology, catalysis, and nanotechnology. ${ }^{3-6}$ In combination with bright synchrotron radiation (SR) sources, XES can be employed to study core-to-core (CtC) or valence-to-core (VtC) transitions. ${ }^{7-9}$ While XES spectra of $3 \mathrm{~d}$ transition elements have been widely studied, ${ }^{10}$ XES spectra of $4 \mathrm{~d}$ elements are not widely reported. ${ }^{1-13}$

The exploration of the spectroscopic X-ray emission signatures of different metal compounds requires high-energy resolution measurements. To measure X-rays emission spectra with a required energy resolution comparable to the lifetime broadening of core holes or better ( a few electron volts), the use of crystal-based $\mathrm{X}$-ray spectrometers is mandatory. Wavelength-dispersive X-ray spectrometers use the diffraction of photons on a crystal to spread 
out the incident radiation with respect to its wavelength. Only photons fulfilling the Bragg law ${ }^{14}$ are diffracted by the crystallographic planes. The accurate measurement of the Bragg angle of the diffracted photons provides precise information on the shape and energy position of the emission lines and thus on the chemistry of the investigated element of interest. Wavelength-dispersive devices are generally divided into reflection (Bragg)- and transmission (Laue)-type spectrometers. In the Bragg-type geometry (e.g., Johann, ${ }^{15}$ Johansson, ${ }^{16}$ and von Hamos ${ }^{17,18}$ spectrometers) the diffraction planes are parallel to the crystal surface (with the exception of the Johansson geometry, in which the crystal is bent and additionally grinded to follow the Rowland radius). In the Lauetype geometry (e.g., Cauchois ${ }^{19}$ and DuMond ${ }^{20}$ spectrometers) the diffraction planes are normal to the bent crystal surface (Fig. 1).

According to the Bragg law, ${ }^{14}$ an increase of the incident X-ray energy leads to a decrease of the Bragg angle. Due to geometrical limitations related to the spectrometer design [as at small Bragg angles, $\theta_{B}$, the energy resolution $\Delta E$ worsens $\left(\Delta E \sim \cot \theta_{B}\right)$ ], the minimum Bragg angles attainable by reflection-type spectrometers have to be higher than $20^{\circ}$. Additionally, with an increase of the incident X-ray energy, the penetration depth of the X-rays in the crystal increases and, for instance, for Si-based materials, the penetration depth changes nonlinearly from $100 \mu \mathrm{m}$ at $10 \mathrm{keV}$ to $3000 \mu \mathrm{m}$ at $30 \mathrm{keV} .^{21,22}$ This increased penetration depth leads to a broadening of the measured X-ray emission lines and consequently to a worsening of the energy resolution. Furthermore, the efficiency of reflection-type spectrometers rapidly decreases with increasing $\mathrm{X}$-ray energy above $10 \mathrm{keV} .{ }^{21,22}$ Therefore, reflection-type crystal spectrometers are generally used for X-ray energies up to $15 \mathrm{keV}$, with few exceptions. For example, a Johann-type spectrometer was used for studies at the Mo $K$-edge $(20 \mathrm{keV})$ using the ninth diffraction order of $\mathrm{Ge}(111)$ crystals. ${ }^{23}$ Note that this was possible because, according to the XOP2.4 code, ${ }^{24-26}$ the integral reflectivity of $\mathrm{Ge}(999)$ is only 46 times smaller than that of $\mathrm{Ge}(111)$.

In the Laue geometry, the X-ray photons have to be transmitted through the crystal to reach the detector placed behind the crystal (Fig. 1). This causes partial absorbance of the diffracted beam, where the absorbance decreases, however, with increasing X-ray energy. A typical thickness of crystals used in transmission-type X-ray spectrometers is $0.5 \mathrm{~mm}$. For $0.5 \mathrm{~mm}$ thick $\mathrm{Si}$ and $\mathrm{SiO}_{2}$ crystals, the transmission of $15 \mathrm{keV} X$-rays amounts to $30 \%$ and $50 \%$, respectively. ${ }^{21,22}$ Consequently, Bragg-type and Laue-type crystal spectrometers can be considered as complementary devices.

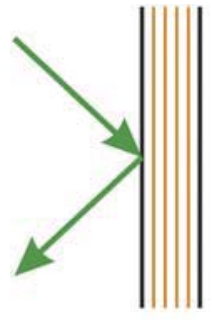

Bragg

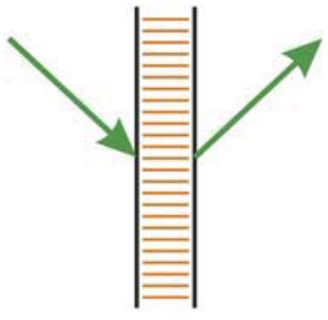

Laue
FIG. 1. Schematic drawing showing the orientation of the X-ray crystallographic planes in the reflection (Bragg) and transmission (Laue) geometry.
The Cauchois ${ }^{19}$ and DuMond ${ }^{20}$ geometries are the most commonly used setups for transmission-type spectrometers. They both require a cylindrically curved crystal with a radius of curvature $\left(R_{C}\right)$ that is equal to the diameter $\left(2 R_{R}\right)$ of the focal Rowland circle (Fig. 2). In the Cauchois geometry, the detector is placed on the Rowland circle, while the source is located outside of the Rowland circle on the other side of the crystal. The DuMond geometry is the reverse of the Cauchois geometry. Here, the source (sample) is placed on the Rowland circle while the detector is placed outside the Rowland circle, as schematically shown in Fig. 2. The Cauchois geometry allows the focusing in the Rowland plane of photons from extended X-ray sources. ${ }^{27,28}$ In this case, photons of different energies are focused, at different positions on the focal (Rowland) circle so that a movable small size detector or a positionsensitive detector should be scanned along the Rowland circle to obtain an X-ray emission spectrum. The Cauchois geometry is thus useful for X-ray studies using large size X-ray sources. In contrast, the DuMond geometry allows analyzing point-like sources

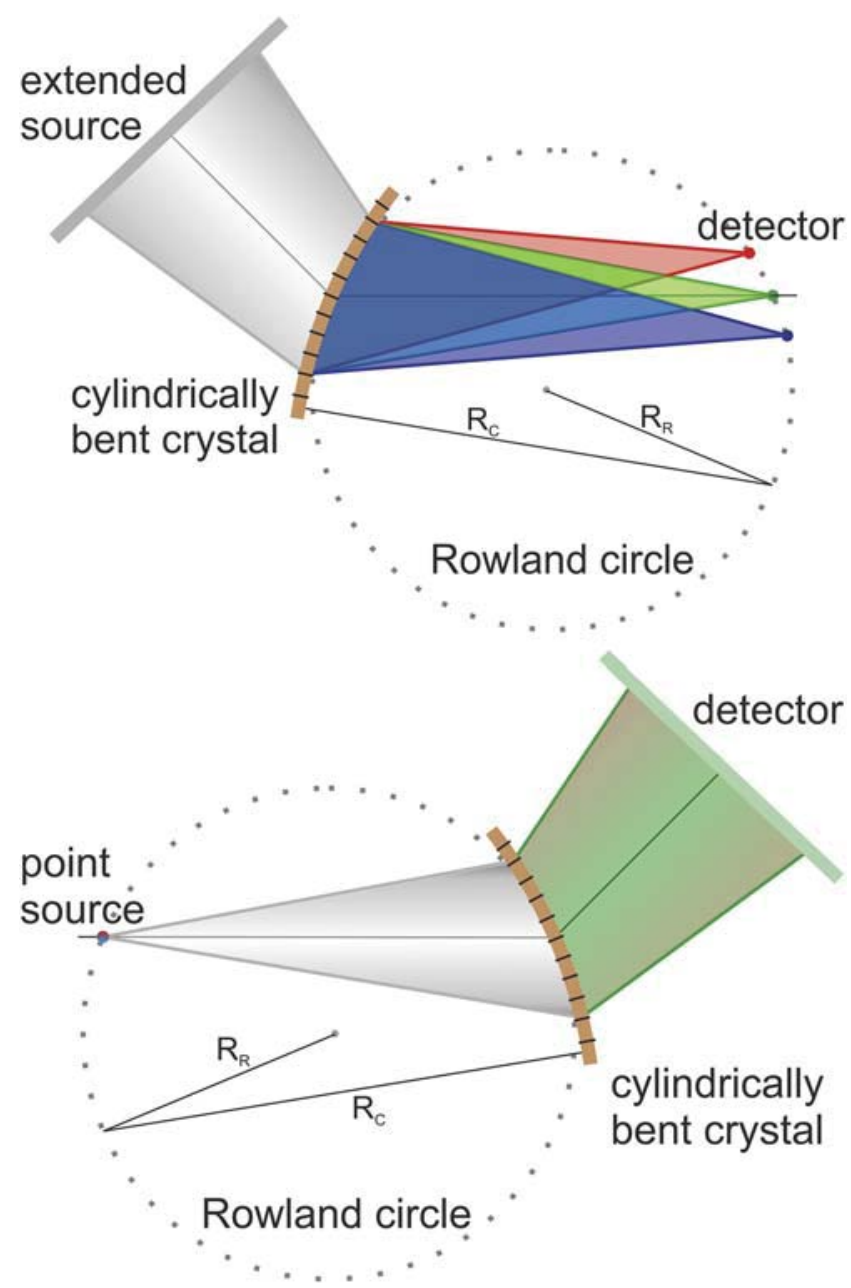

FIG. 2. Schemes of Laue-type spectrometers in the Cauchois (top panel) and DuMond (bottom panel) geometry. 
placed on the focal circle. For a given source-crystal orientation, the DuMond spectrometer operates as a monochromator, in which a wide crystal area diffracts only one wavelength per diffraction order.

Since the first DuMond spectrometer ${ }^{20}$ was developed, many devices based on this geometry have been constructed. DuMond spectrometers were used to measure the $\gamma$-rays produced in nuclear reactions $\mathrm{s}^{29,30}$ as well as the X-rays emitted as a result of the radiative Auger effect, ${ }^{31}$ the multiple inner-shell ionization, ${ }^{32}$ the radiative decay of mesonic, ${ }^{33,34}$ muonic $^{35,36}$ atoms, and of highly charged high-Z ions. ${ }^{37}$ Transmission-type spectrometers were also used at SR facilities to study Compton scattering with high energy resolution. Using $115 \mathrm{keV}$ photons, a DuMond spectrometer with a Rowland circle radius of $3650 \mathrm{~mm}$ reached an energy resolution of $80 \mathrm{eV}$ at that energy. ${ }^{38,39}$ Measurements of the Compton profile, for photons with an energy of $90 \mathrm{keV}$ and with an energy resolution of $90 \mathrm{eV}$, were also performed by Hiraoka et al. ${ }^{40}$ using a spectrometer with a crystal bending radius of $1.6 \mathrm{~m}$. Furthermore, a transmissiontype spectrometer with a Rowland circle diameter of $965 \mathrm{~mm}$ was employed for measurements of the resolution of the $K \alpha_{1,2}$ lines of Ho (energies of $47.5 \mathrm{keV}$ and $46.7 \mathrm{keV}$, respectively) which amounted to $69 \mathrm{eV}^{41}$

In this paper, we present a high-resolution Laue-type X-ray spectrometer which was designed for measurements with synchrotron light and is installed at the SuperXAS beamline of the Swiss Light Source (SLS). Because of the small X-ray spot size on the sample at the SuperXAS beamline (typically $~ 100 \times 100 \mu \mathrm{m}^{2}$ ), the DuMond geometry, which is designed for point-like X-ray sources, ${ }^{27,28}$ was chosen. The design of the spectrometer was inspired by a high-resolution DuMond crystal spectrometer developed at the University of Fribourg, Switzerland, which is used for in-house measurements. ${ }^{42}$ The spectrometer of Fribourg is equipped with a $0.5 \mathrm{~mm}$ thick quartz crystal having a radius of curvature of $3150 \mathrm{~mm}$. It is operated in the modified DuMond slit geometry. In this geometry, a narrow rectangular slit is placed on the focal circle at a fixed position and acts as an effective source of radiation, while the target/sample is placed behind the slit. A scintillation detector records the X-rays coming from the sample and diffracted by the crystal. For the first order of diffraction, the FWHM instrumental resolution of the spectrometer of Fribourg is $40.3 \mathrm{eV}$ for the Gd $K \alpha_{1}$ $\mathrm{X}$-ray line $(42.996 \mathrm{keV})$ and $6.6 \mathrm{eV}$ for the Mo $K \alpha_{1} \mathrm{X}$-ray line $(17.479 \mathrm{keV})$. Furthermore, the use of this spectrometer for laboratory-based XAS measurements was probed successfully, using an X-ray tube to produce the continuous energy X-ray beam. ${ }^{42}$

The DuMond spectrometer presented in this paper is equipped with a dedicated crystal bender and a two-dimensional single photon counting pixel detector. The spectrometer was designed for high energy-resolution XES measurements in the $15-26 \mathrm{keV}$ energy range with envisioned application for XES measurements of valence-tocore transitions of $4 \mathrm{~d}$ elements. Such measurements represent a powerful tool for the chemical characterization of materials. A correct interpretation of the weak VtC X-ray emission spectra requires, however, high energy resolution measurements with an experimental resolution better than the natural $K$-shell core-hole lifetime broadening of the initial state, which, for example, for $\mathrm{Ru}$ is $5.33 \mathrm{eV} .{ }^{43}$ As the first application of the novel instrument, the VtC spectra of several $\mathrm{Ru}$ compounds were measured. These results are presented at the end of the paper.

\section{EXPERIMENTAL FACILITY}

\section{A. SuperXAS beamline}

The Swiss Light Source (SLS, Villigen-PSI) operates in the top up mode at a ring electron current of $400 \mathrm{~mA}$ and an energy of $2.4 \mathrm{GeV}$. The SuperXAS beamline is located at a $2.9 \mathrm{~T}$ supercooled bending magnet source, with a critical energy of $11.9 \mathrm{keV}$. X-rays from the bending magnet can be collimated by different mirror coatings such $\mathrm{Rh}, \mathrm{Pt}$, and $\mathrm{Si}$ (in the measurements presented in this paper only the Pt coating was used). A channel-cut crystal monochromator with a pair of $\mathrm{Si}(111)$ and a pair of $\mathrm{Si}(311)$ crystals is located downstream of the collimating mirror. This monochromator allows us to select the photon energy from $4.0 \mathrm{keV}$ to $35 \mathrm{keV}$ with an intrinsic energy resolution $\left(\Delta E_{\text {intr }} / E_{0}\right)$, for the here used $\operatorname{Si}(111)$ crystal pair of $1.4 \times 10^{-4}$ and a flux of $10^{11}-10^{12}$ photons $/ \mathrm{s}^{44}$ The monochromatic X-ray beam can be focused by a Rh or a Pt coated toroidal mirror located after the channel cut monochromator to a spot size of $100 \times 100 \mu \mathrm{m}^{2}$ on the sample position. ${ }^{45}$

The SuperXAS beamline is equipped with two Bragg-type X-ray spectrometers, i.e., a five-crystal Johann spectrometer ${ }^{46}$ and a segmented-crystal von Hamos spectrometer. ${ }^{47}$ Both of them work in Bragg geometry (reflection type), and are therefore inefficient at X-ray energies above $\sim 15 \mathrm{keV}$. The DuMond spectrometer,

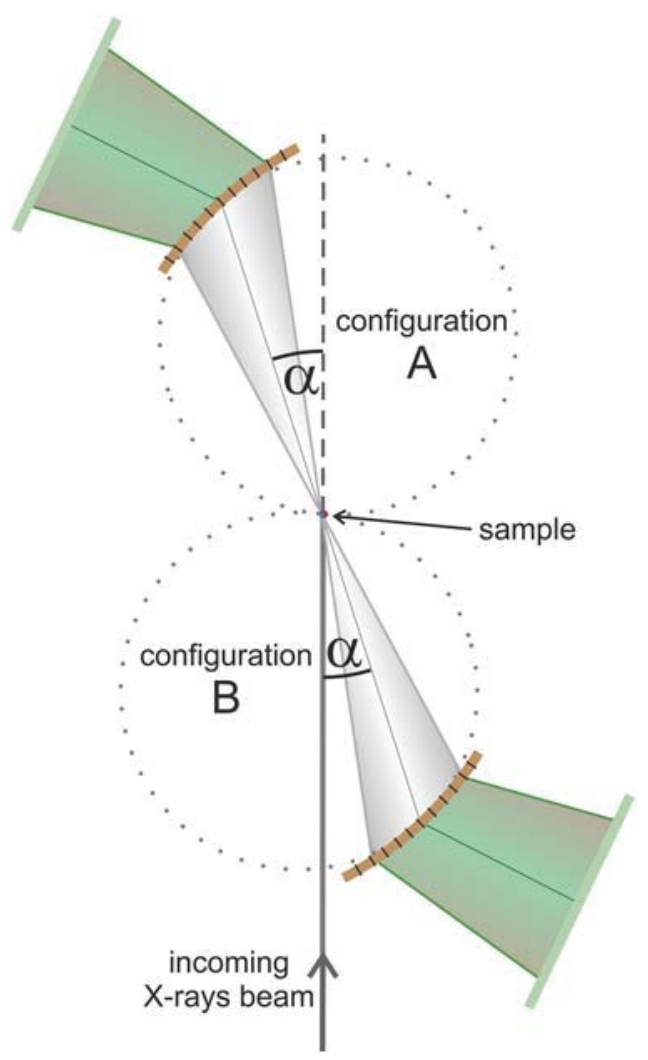

FIG. 3. Scheme of the two possible configurations of the DuMond spectrometer at the SuperXAS beamline. Configuration A: forward emitted X-rays. Configuration B: backward emitted X-rays. 
presented here, can be installed in two different configurations at the SuperXAS beamline (Fig. 3). In configuration "A," the X-rays emitted by the sample in the forward direction are measured. This setting requires the use of an effective shielding to reduce the background from the scattered primary photon beam. In configuration " $\mathrm{B}$," the $\mathrm{X}$-ray radiation from the sample emitted in the backward direction is measured.

The distance between the sample and the crystal is determined by the DuMond geometry. Due to geometrical constraints originating from the spectrometer design (e.g., selected size of the Rowland circle), the angle $\alpha$ between the X-ray beam and the spectrometer sample-to-crystal-center direction (Fig. 3) must be smaller than $10^{\circ}$. The measurements have shown that the peak-to-background $(\mathrm{p} / \mathrm{b})$ ratio is about three times better for the configuration "B" (Fig. 4) due to reduction of scattering of the primary beam. For this reason, this geometry was chosen for all following experiments.

\section{B. Spectrometer design}

The DuMond spectrometer designed for use at the SuperXAS beamline of the SLS is foreseen to measure X-ray emission lines between $15 \mathrm{keV}$ and $26 \mathrm{keV}$. The spectrometer consists of a cylindrically bent crystal for X-ray diffraction and a position sensitive single photon counting Pilatus 100K-S 2D-detector. ${ }^{48}$ The spectrometer is mounted on a moveable, motorized tower (Fig. 5). This tower can be moved independently in the dispersion plane (horizontal $x y$-plane) as well as in the vertical direction ( $z$-axis). This is realized by using three linear motors. Additionally, the tower is equipped with a $\theta-2 \theta$ stage, which consists of two rotation motors, for the crystal and the detector, respectively. These motors are mounted in such a way that the concentricity of both rotation axes is ensured. To set the desired crystal-detector distance $(d)$ one more linear stage is used. The stepping resolution of the linear and rotational motors is $1 \mu \mathrm{m}$ and $0.001^{\circ}$, respectively, which enables the necessary precision of the alignment of the crystal and detector.

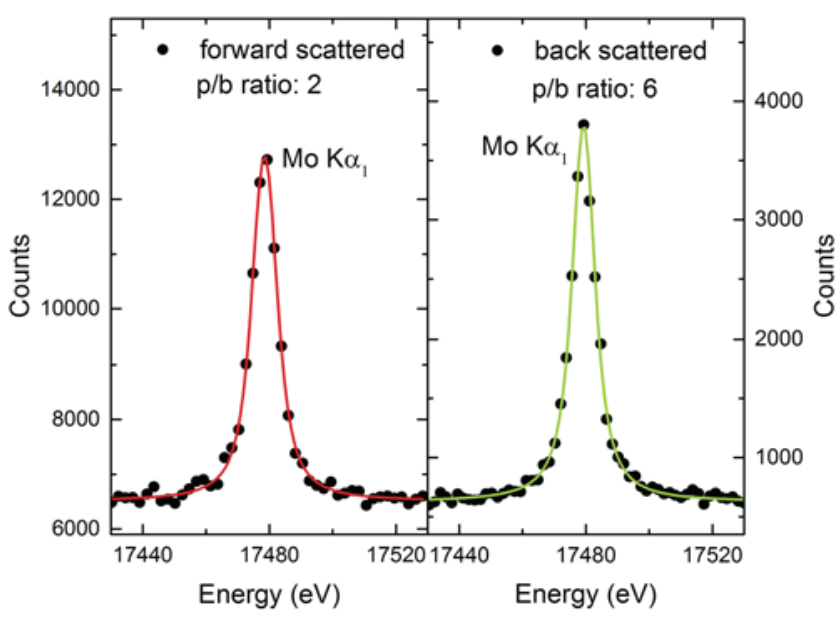

FIG. 4. The Mo $K \alpha_{1}$ X-ray emission line measured using the two different configurations shown in Fig. 3. Left panel: forward configuration ("A"). Right panel: backward configuration (" $\mathrm{B}$ "). The peak-to-background ( $\mathrm{p} / \mathrm{b}$ ) ratio is about 2 for the configuration " $\mathrm{A}$ " and about 6 for the configuration " $\mathrm{B}$ ".

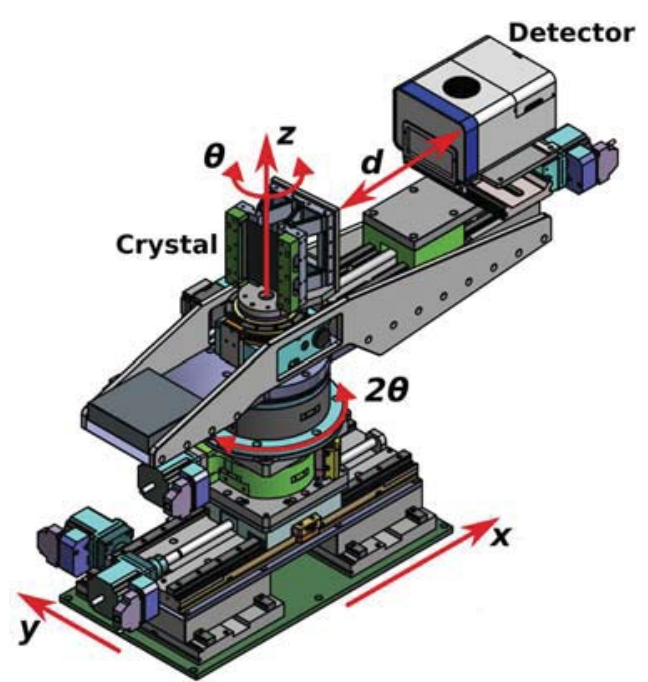

FIG. 5. Technical drawing of the DuMond spectrometer installed at the SuperXAS beamline.

The crystal stage and the linear $x$-axis stage are equipped with encoders.

In the DuMond geometry, the distance between the source (sample/target) and the crystal $\left(L_{B}\right)$ is given by $L_{B}=R_{C} \cos \theta_{B}$, where $R_{C}$ and $\theta_{B}$ are the crystal bending radius and Bragg angle, respectively, as schematically depicted in Fig. 6. A change of the Bragg angle $\left(\theta_{B}\right)$ modifies therefore the distance $\left(L_{B}\right)$, whereas the crystal-to-detector distance $(d)$ remains unchanged. If needed,

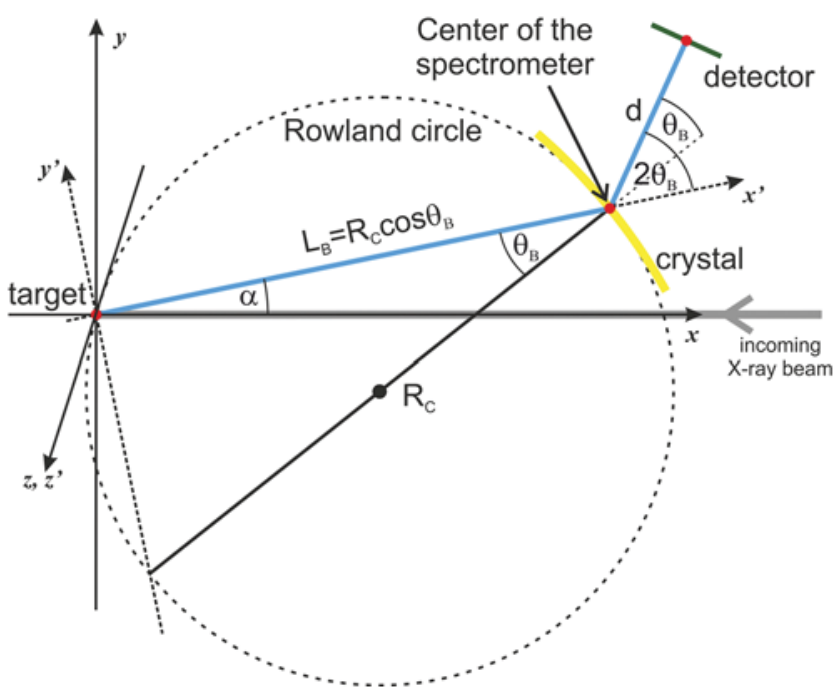

FIG. 6. Geometry of the DuMond spectrometer in the backward configuration showing the point-like source of radiation (sample/target), the bent crystal, and the detector. The crystal radius of curvature $R_{C}$ is equal to the diameter of the Rowland circle. $\theta_{B}$ is the Bragg angle and, $\alpha$ the angle between the synchrotron radiation beam and the source/sample-to-crystal direction. The $(x y z)$ coordinate system is related to the beamline coordinate system, while the $\left(x^{\prime} y^{\prime} z^{\prime}\right)$ coordinate system is related to the DuMond spectrometer. 
however, the latter can be changed independently. The spectrometer design allows setting this crystal-to-detector distance in the range of 200-500 $\mathrm{mm}$. The sample manipulator is independent from the spectrometer tower and is mounted along the axis of the beam $(x$-axis).

The sample is positioned at $90^{\circ}$ relative to the beam direction. For a given Bragg angle, the coordinates of the spectrometer with respect to the sample are calculated according to the DuMond geometry using the following expressions: $x=L_{B} \cos \alpha$ and $y=L_{B} \sin \alpha$, where $\alpha$ is the angle between the incoming beam and the spectrometer source-to-crystal direction (Fig. 6), and $L_{B}$ is given by the formula presented in the previous paragraph. The coordinates $x$ and $y$ are set by using the corresponding linear stages. A laser-line leveler is used for the rough alignment of the spectrometer height and to align the height of the crystal center on the axis of the incoming photon beam. The final alignment of the spectrometer position is performed with X-rays. In this final alignment stage, the spectrometer position is adjusted to maximize the signal and minimize the width of the $\mathrm{X}$-ray emission spectra recorded on the $2 \mathrm{D}$-detector.

\section{Crystal and its bender}

The spectrometer is equipped with a $0.5 \mathrm{~mm}$ thick square crystal. The diffraction planes of the crystal are perpendicular to the front surface of the crystal. The crystal size is $100 \times 100 \mathrm{~mm}^{2}$, and because of the clamps of the bender, the effective reflecting area is $70 \times 100 \mathrm{~mm}^{2}$ (width $\times$ height). A dedicated crystal bender allowing to vary the crystal bending radius from $1.3 \mathrm{~m}$ to $2.5 \mathrm{~m}$ is employed. Based on Monte Carlo ray-tracing calculations of the characteristics of the DuMond spectrometer for different crystals, a Si(111) crystal with a thickness of $0.5 \mathrm{~mm}$ was chosen. A more detailed description of the Monte Carlo simulations is presented in Sec. III.

The crystal bender is based on the design of a KB mirror bender of the Deutsches Elektronen-Synchrotron (DESY) Institute. It is constructed in such a way that it prebends the crystal to a bending radius of $1.3 \mathrm{~m}$. The crystal is mounted in the crystal bender (Fig. 7)

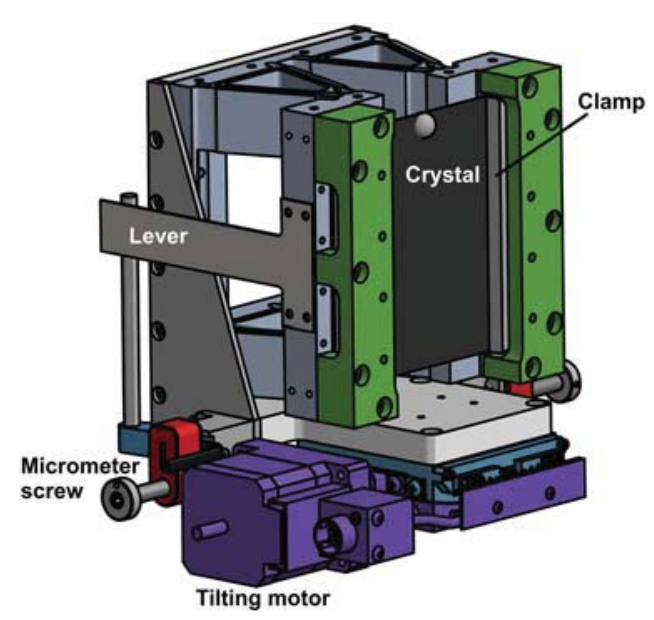

FIG. 7. Technical drawing of the crystal bender with the crystal mounted between the bender blocks and clamps. The two micrometer screws used to apply the bending torque are also shown. by means of two clamps, which press the vertical sides of the crystal to flat bender blocks. These blocks are arranged symmetrically with respect to the center of the bender. Levers which are connected to two independent micrometer screws are fixed to the clamps. The bending torque is applied to the levers by pushing them via the micrometer screws pivots. The design of the crystal bender imposes limitations on the maximum Bragg angle that can be achieved. The distance between the crystal and the rear part of the bender $(8 \mathrm{~cm})$ and the width of the bender aperture $(7 \mathrm{~cm})$ allow to set the Bragg angle to a maximum value of $26^{\circ}$ (Fig. 8). Above $26^{\circ}$, the bender starts to partially block the diffracted beam (shadowing effect). For the $\mathrm{Si}(111)$ crystal and the 3 rd order of reflection, this maximum angle corresponds to a minimum X-ray energy of $13.5 \mathrm{keV}$.

The quality of curvature of the silicon crystal was tested using a CNC 3D-Coordinate Measuring Machine by mapping the crystal surface, mounted in the bender, at 70 different points. Besides fluctuations of the bending radius, the measurements showed some waviness of the crystal surface in the direction perpendicular to the bending plane. This unavoidable waviness, which affects the energy resolution of the spectrometer, is a consequence of Hooke's law according to which the curvature in one direction induces a curvature of opposite sign in the perpendicular direction. ${ }^{49}$

The shape of the $\mathrm{Si}(111)$ crystal surface was measured for bending radii of $1.3 \mathrm{~m}$ and $2.5 \mathrm{~m}$. Figure 9 shows the results of the measurement of the shape and waviness of the crystal bent to a radius of $2.5 \mathrm{~m}$. The maximum deviations, i.e., the differences between the highest and lowest distortions, were found to be located in the center of the bent crystal (solid straight line in the bottom panel of Fig. 9). Maximum deviations of the curvature of $20 \mu \mathrm{m}$ and $15 \mu \mathrm{m}$ were obtained for curvature radii of $1.3 \mathrm{~m}$ and $2.5 \mathrm{~m}$, respectively. Hence, these measurements suggest that the deviations from the ideal cylindrical curvature decrease with increasing bending radius.

\section{Detector}

The spectrometer is equipped with an air-cooled Pilatus $100 \mathrm{~K}-\mathrm{S} 2 \mathrm{D}$-detector. ${ }^{48}$ The use of a two-dimensional detector permits to optimize the signal diffracted by the crystal. The detector is composed of a $1 \mathrm{~mm}$ thick monolithic silicon sensor array of pndiodes with a pixel size of $172 \times 172 \mu \mathrm{m}^{2}$. The matrix has a size of $487 \times 195$ pixels and covers an active area of $83.8 \times 33.5 \mathrm{~mm}^{2}$. The increased thickness ( $1 \mathrm{~mm}$ vs $450 \mu \mathrm{m}$ ) of the silicon sensor, results in a better quantum efficiency at the higher X-ray energies. Using here a single photon counting detector with a CdTe sensor would

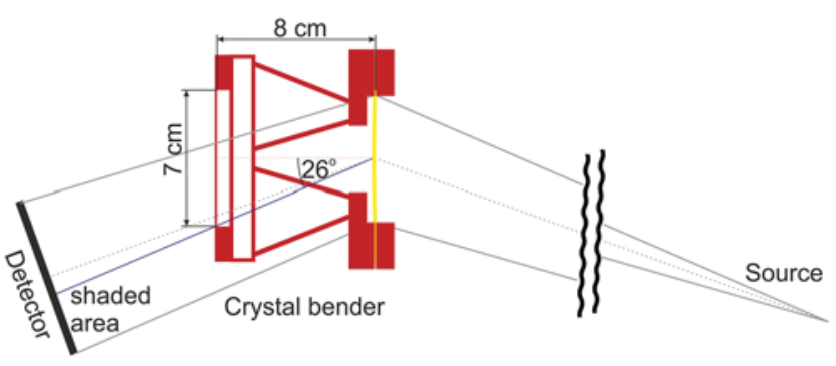

FIG. 8. Scheme of the crystal bender showing the shadowing effect. 

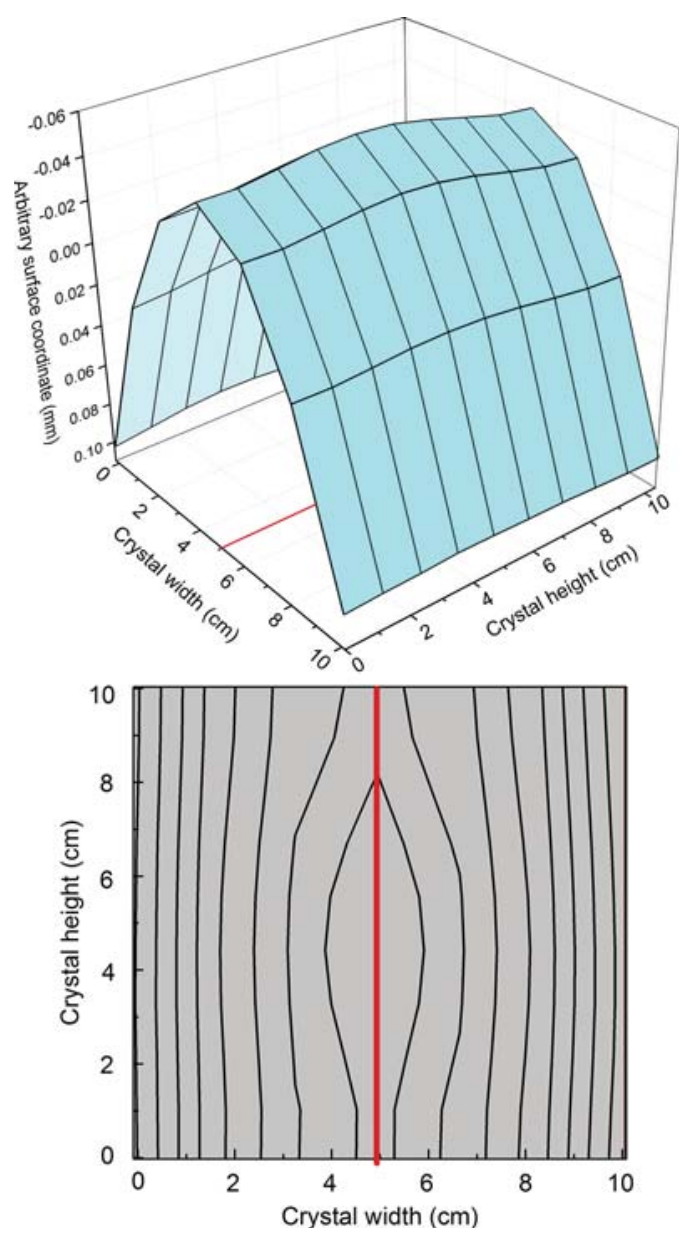

FIG. 9. Shape of the Si(111) crystal surface bent to a radius of $2.5 \mathrm{~m}$. The shape was measured using a CNC 3D-Coordinate measuring machine (top panel). Projection of the measured crystal surface on the 2D plane (bottom panel).

improve the quantum efficient at least twice. The maximum counting rate of the Pilatus detector amounts to $2 \times 10^{6} \mathrm{X}$-ray/s/pixel. This $\mathrm{X}$-ray detector operates in the so-called "single photon counting" mode. Each pixel has its own amplifier and counting circuit. The method of extracting the signal from the $2 \mathrm{D}$ image is described in Sec. IV.

\section{MONTE CARLO RAY-TRACING SIMULATION}

A straightforward procedure to determine the main characteristics of an X-ray spectrometer and to better understand the optical properties of the designed device is a ray-tracing simulation approach. The Monte Carlo method is especially well suited and efficient for simulations where, due to the complexity of the problem, a fully analytical treatment is impractical. Therefore, the Monte Carlo X-ray-tracing code, ${ }^{50}$ which was developed to simulate the intensity distribution of diffracted X-rays on a detector, for various $\mathrm{X}$-ray spectrometer geometries and crystals, was adapted here for the DuMond $\theta-2 \theta$ configuration (Fig. 6). This code called DMD
$\mathrm{X}$-ray-tracing is written in $\mathrm{C}++$. The code tracks the trajectory of each photon emitted randomly at the X-ray source (sample) and checks whether this photon is diffracted by the crystal and registered by the detector. In the case that the photon reaches the crystal surface, the diffraction process described by the crystal rocking curve (from XOP2. $4^{24-26}$ ) using dynamical theory ${ }^{51,52}$ is considered. The photon diffracted by the crystal is then traced on its way to the $2 \mathrm{D}$ detector. The X-ray source considered is defined by the conditions of the discussed experiment where the photons are emitted from the sample illuminated by SR. The simulation starts from generation of six random numbers, namely, two photon emission angles $\xi$ and $\varphi$ in a spherical coordinate system, three coordinates $\left(x_{s}^{\prime}, y_{s}^{\prime}, z_{s}^{\prime}\right)$ of a point of photon emission from the sample, and the photon energy $E$ taking into account a natural linewidth of the transition given by a Lorentz energy distribution. In these simulations, the uniform random number generators based on a Mersenne Twister algorithm ${ }^{53}$ and transformations were used to generate the random numbers having desired distributions. In this setting a direction of the emitted photon can be expressed in terms of two angles $\xi$ and $\varphi$ by the unit vector $\vec{S}=\left[S_{X}^{\prime}, S_{Y}^{\prime}, S_{Z}^{\prime}\right]=[\cos \xi, \sin \xi \cos \varphi, \sin \xi \sin \varphi]$. Each photon emitted from the X-ray source is traced from the source to crystal. A possible diffraction point of the photon at the crystal is determined by solving the system of equations of a straight line of the photon path and a crystal surface which is cylindrically bent with a radius $R_{C}$

$$
\left\{\begin{array}{l}
x^{\prime}=x_{s}^{\prime}+S_{X}^{\prime} \cdot t, \\
y^{\prime}=y_{s}^{\prime}+S_{Y}^{\prime} \cdot t, \\
z^{\prime}=z_{s}^{\prime}+S_{Z}^{\prime} \cdot t, \\
\left(x^{\prime}-x_{c}^{\prime}\right)^{2}+\left(y^{\prime}-y_{c}^{\prime}\right)^{2}=R_{C}^{2}, \\
h_{1}<z^{\prime}<h_{2},
\end{array}\right.
$$

where $\left(x_{c}^{\prime}, y_{c}^{\prime}, z_{c}^{\prime}\right)$ is the center of the axis of a cylinder describing the crystal surface, $h_{1}, h_{2}$ is the crystal size in the $z$-direction and the photon trajectory is described parametrically by $t$. Additionally, the traced diffraction events had to be confined to the assumed dimensions of the crystal. This system of equations describes an intersection point $\left(x_{k}^{\prime}, y_{k}^{\prime}, z_{k}^{\prime}\right)$ on a crystal surface. For each calculated diffraction point $\left(x_{k}^{\prime}, y_{k}^{\prime}, z_{k}^{\prime}\right)$ on the crystal surface, the angle $\theta$ between photon direction and crystallographic planes is calculated. The difference $\Delta \theta=\theta-\theta_{B}$ determines the probability of $\mathrm{X}$-ray diffraction according to the dynamical theory described by the diffraction profile called crystal rocking curve. The $\theta_{B}$ is the Bragg angle binding the geometry of a crystal with the wavelength of incident photons as described by Bragg's law

$$
m \lambda=2 d_{h k l} \sin \theta_{B},
$$

where $m$ denotes the order of diffraction, $\lambda$ is the X-ray wavelength, and $d_{h k l}$ the spacing constant of the diffraction planes $(h k l)$. Additionally, the crystal deformation described by a Gaussian function is taken into account. Parameters of this profile are taken from measurement of mapping the crystal surface (see Sec. II C). The photon diffracted from the crystal with a direction $\vec{K}=\left[K_{X}^{\prime}, K_{Y}^{\prime}, K_{Z}^{\prime}\right]$ was traced on their way to the X-ray detector. The point $\left(x_{d}^{\prime}, y_{d}^{\prime}, z_{d}^{\prime}\right)$ of recording the photon on the detector was calculated by solving the system of equations describing the point of intersection of a straight 
line diffracted photon path and a plane representing the detector surface

$$
\left\{\begin{array}{l}
x^{\prime}=x_{k}^{\prime}+K_{X}^{\prime} \cdot t, \\
y^{\prime}=y_{k}^{\prime}+K_{Y}^{\prime} \cdot t, \\
z^{\prime}=z_{k}^{\prime}+K_{Z}^{\prime} \cdot t, \\
\Upsilon x^{\prime}+\Phi y^{\prime}+\Psi z^{\prime}+\Omega=0,
\end{array}\right.
$$

where $\Upsilon, \Phi, \Psi, \Omega$ are the parameters describing the location of the detector surface for a given Bragg angle.

The diffraction of X-rays on crystals is more complex than described by the ordinary Bragg law of Eq. (1). The Bragg law assumes that the X-rays are singly reflected at the angle of diffraction $\theta_{B}$ on the crystal planes without refraction on the crystal surface and without absorption.

The dynamical theory of diffraction, in the treatment of Darwin, ${ }^{51,52}$ accounts for the effects of X-ray refraction, absorption, and multiple reflections in a macroscopic perfect crystal and leads to a corrected form of the Bragg angle. ${ }^{54}$ In this approach, the diffracted photons are scattered with a certain probability in a narrow angular range of $\theta$ near the Bragg angle $\theta_{B}$. This effect is accounted for by the crystal rocking curve which describes the angular distribution of $\mathrm{X}$-rays diffracted from the crystal surface and is usually expressed as a function of relative angle $\Delta \theta=\theta-\theta_{B}$ characterized by some finite width. This dynamical theory of diffraction approach was used to determine the main effects influencing the characteristics of the DuMond spectrometer.

All contributions to the instrumental energy resolution of the DuMond spectrometer were simulated taking into account the measured characteristics of the source/target and the crystal, including deformation caused by its bending (see Sec. II C). The rocking curves were calculated using the XCRYSTAL module with selected bent crystal models of the XOP2.4 $4^{24-26}$ code and dynamical theory of diffraction. Figure 10 shows the rocking curves of the $\operatorname{Si}(111)$ and $\mathrm{SiO}_{2}(110)$ crystals calculated for the $\mathrm{Ru} K \alpha_{1}$ line $(19.279 \mathrm{keV})$.

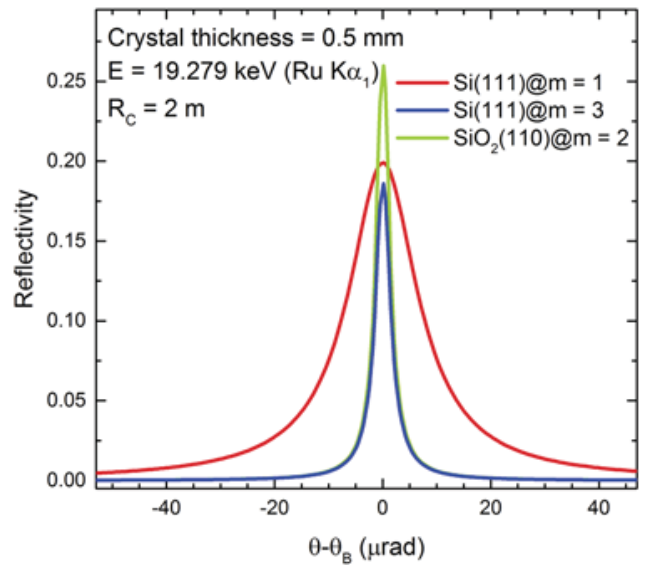

FIG. 10. Calculated rocking curves for transmission (Laue) geometry for two different crystals bent both to the radius of $R_{C}=2 \mathrm{~m}$ : Si(111) in 1st and 3rd diffraction order, and $\mathrm{SiO}_{2}(110)$ in 2 nd order. The calculations were performed for the $\mathrm{Ru} K \alpha_{1}$ line $(19.279 \mathrm{keV})$ using the XOP $2.4^{24-26}$ code and the dynamical theory of diffraction.
The crystal rocking curves for the Laue case are symmetrical and centered at Bragg angle given by Eq. (1). The rocking curves of the $\mathrm{Si}(111)$ crystal, calculated for the 1st and 3rd orders of reflection, show that the curve width decreases with increasing order of reflection. The widths of the calculated crystal rocking curves for the silicon crystal in the 3rd order of reflection and the quartz crystal in the 2nd order are comparable, but the reflectivity at the peak is higher for the quartz crystal than for the silicon one. All calculations of crystal rocking curves were done assuming perfect crystals.

For a DuMond type spectrometer, the energy resolution results from the convolution of various parameters ${ }^{55}$ including the natural broadening of the emission line $(\Gamma)$, the X-ray source size, the uncertainty of the crystal bending, the crystal deformation, and the crystal size. Because the DuMond crystal spectrometer works in $\theta-2 \theta$ configuration, where the $\mathrm{X}$-ray energy is selected by scanning the Bragg angle, the pixel size of the detector has no direct influence on the energy resolution, and therefore, this parameter was not included in the simulations. Using the Monte Carlo ray-tracing simulations, the instrumental energy resolution was simulated, as an example, for the $\mathrm{Ru} K \alpha_{1} \mathrm{X}$-ray line. The crystal rocking curve shape, simulated for a point-like X-ray source and a small crystal size with perfect shape (without deformation) contribute to an instrumental energy resolution of $0.81 \mathrm{eV}$ (Table I). The effect of enlarging the X-ray source size on the instrumental energy resolution simulated for the DoMond spectrometer is presented in Fig. 11. The simulations were performed for an X-ray energy corresponding again to the $\mathrm{Ru} K \alpha_{1}$ line, for a $\mathrm{Si}(111)$ crystal with a bending radius of $2 \mathrm{~m}$ and the $3 \mathrm{rd}$ order of reflection. Contributions of $1.25 \mathrm{eV}, 2.28 \mathrm{eV}$, and $3.30 \mathrm{eV}$ were obtained for an X-ray source size of $50 \times 50,100 \times 100$, and $150 \times 150 \mu \mathrm{m}^{2}$, respectively. The contribution of the crystal size in the dispersion direction $(100 \mathrm{~mm})$ was also simulated. A value of $0.63 \mathrm{eV}$ was found. This contribution was simulated for the crystal size of $100 \mathrm{~mm}$ with perfect cylindrical shape, point-like X-ray source and very narrow and symmetrical rocking curve to eliminate the influence of these parameters. This increase of the energy resolution observed for a crystal of large size (in the direction of dispersion) can be explained by geometrical aberrations related to the not fully focusing DuMond geometry (the angle between the incoming radiation and the diffraction planes is slightly different

TABLE I. Simulated contributions of different effects to the instrumental angular resolution $(\Delta \theta)$ and the instrumental energy resolution $(\Delta E)$ of the DuMond spectrometer. The simulations were performed for the Ru $K \alpha_{1}$ line (19.279 keV), a Si(111) crystal and the 3rd diffraction order.

\begin{tabular}{lcc}
\hline \hline Contribution & $\Delta \theta(\mathrm{mdeg})$ & $\Delta E(\mathrm{eV})$ \\
\hline Rocking curve & 0.78 & 0.81 \\
Source size [including natural broadening of the emission line $(\Gamma)]:$ \\
$50 \times 50 \mu \mathrm{m}^{2}$ & 1.16 & 1.25 \\
$100 \times 100 \mu \mathrm{m}^{2}$ & 2.19 & 2.28 \\
$150 \times 150 \mu \mathrm{m}^{2}$ & 3.17 & 3.30 \\
Crystal size $(100 \mathrm{~mm})$ & 0.61 & 0.63 \\
Crystal deformation & 1.17 & 1.22 \\
Total $\left(100 \times 100 \mu \mathrm{m}^{2}\right.$ source size $)$ & 4.54 & 4.73 \\
\hline
\end{tabular}




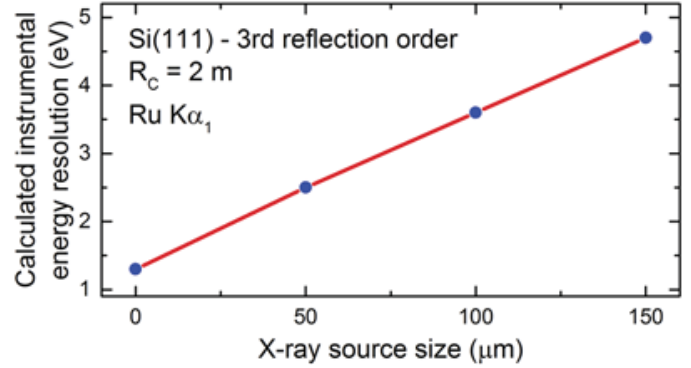

FIG. 11. Variation of the instrumental energy resolution of the DuMond spectrometer as a function of the source size (in direction of dispersion) as predicted by Monte Carlo simulations.

at the edges of the crystal than in the center, the difference growing with the distance to the center). Additionally, the contribution of the crystal deformation, which is the cost of crystal bending and final crystal size, was simulated for a point-like X-ray source. For this effect, the contribution of $1.22 \mathrm{eV}$ was found. For the discussed settings, the simulated total instrumental energy resolution of the DuMond spectrometer amounts to $4.73 \mathrm{eV}$ for a $\mathrm{Si}(111)$ crystal in 3rd diffraction order. The simulated contributions of the different parameters influencing the broadening are summarized in Table I. It is important to know, that the parameters listed in Table I, which contribute to the instrumental energy resolution, are not independent. Therefore they values cannot be simply added up, or used in a root sum of the squares. Accordingly, only the Monte Carlo method allows us to test the contribution from each single parameter, as all parameters simultaneously to the instrumental energy resolution.

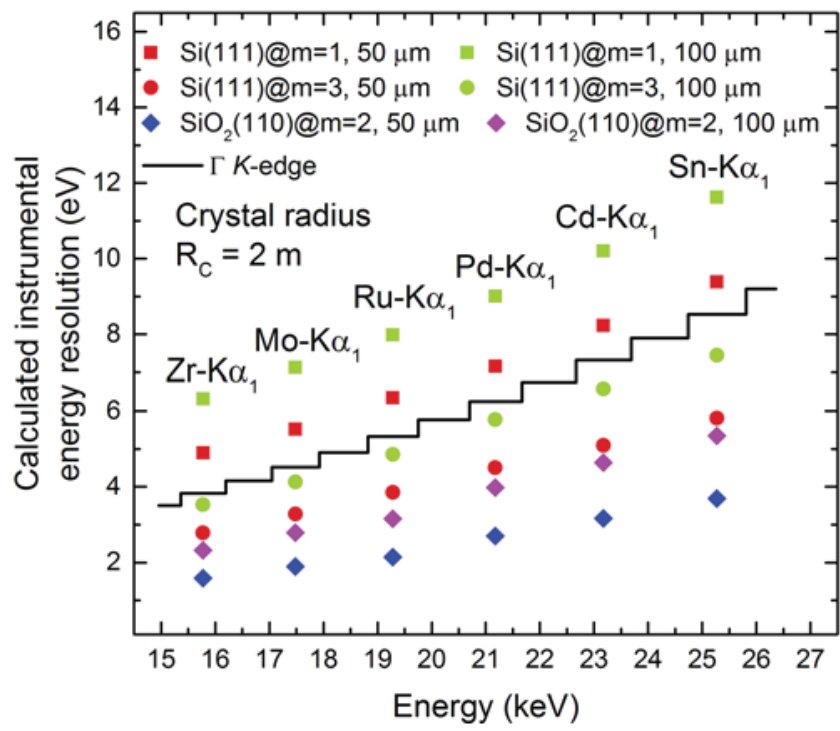

FIG. 12. Comparison of the instrumental energy resolutions of the DuMond spectrometer simulated for different crystals, $\mathrm{X}$-ray energies, $\mathrm{X}$-ray source sizes, and crystal reflection orders $(m=1-3)$. The solid line represents the $K$-shell core-hole lifetime broadening $(\Gamma)$ of elements with $Z=40-50$ reported by Campbell and Papp. $^{43}$
Figure 12 shows the instrumental energy resolution, simulated for different X-ray energies, different X-ray source sizes $(50 \times 50$, $100 \times 100 \mu \mathrm{m}^{2}$ ), crystals $\left(\mathrm{Si}\right.$ and $\left.\mathrm{SiO}_{2}\right)$, and diffraction orders $(\mathrm{m})$. The solid line represents the $K$-shell core-hole lifetime broadening ( $\Gamma)$ in the $16-26 \mathrm{keV}$ range reported by Campbell and Papp. ${ }^{43}$ The simulations show that the instrumental energy resolution for $\mathrm{Si}(111)$ crystal and 1st order of reflection is not sufficient to study VtC X-ray emission spectra with subnatural broadening resolution. These simulations show also that the energy resolution can be improved by measuring the X-ray spectra at higher diffraction orders and also with smaller X-ray source sizes. For example, for the ruthenium $K \alpha_{1}$ line, the instrumental energy resolution for the 3rd order of reflection and the $\mathrm{Si}(111)$ crystal (with a bending radius of $2 \mathrm{~m}$ ) is improved by a factor of 2 with respect to the 1 st order of reflection. Similarly, a reduction of the source size from $100 \times 100 \mu \mathrm{m}^{2}$ to $50 \times 50 \mu \mathrm{m}^{2}$ results in an improvement of the instrumental energy resolution by about $1 \mathrm{eV}$ for the $\mathrm{Ru} K \alpha_{1}$ emission line. In conclusion, the Monte Carlo simulations show that the instrumental energy resolution of DuMond spectrometer, simulated for $\mathrm{Si}(111)$ crystal and 3rd reflection order, is smaller than the K-shell core-hole lifetime broadening. This means that this instrumental energy resolution is sufficient to measure the VtC X-ray emission spectra with subnatural broadening resolution.

\section{SPECTROMETER ENERGY RESOLUTION}

As shown by the MonteCarlo ray-tracing simulations, the main factors influencing the spectrometer energy resolution are the X-ray source size, the bending radius of the crystal, and the precision of the crystal curvature. A large bending radius provides a higher energy resolution, but results in a lower efficiency due to the decrease of the solid angle. Reciprocally, a smaller bending radius leads to an increase of the efficiency but the energy resolution becomes worse.

The instrumental energy resolution of the DuMond spectrometer was experimentally determined by measuring the $K \alpha_{1}$ lines of different elements. Each XES spectrum was recorded by scanning the desired angular range step by step. For every angle (step), the photons registered by the $2 \mathrm{D}$ pixel detector are integrated along the dispersion axis of the detector. Every next angular step produces a new line of events on the 2D map as shown in Fig. 13. On the left side of the selected region of interest (ROI), only the background is

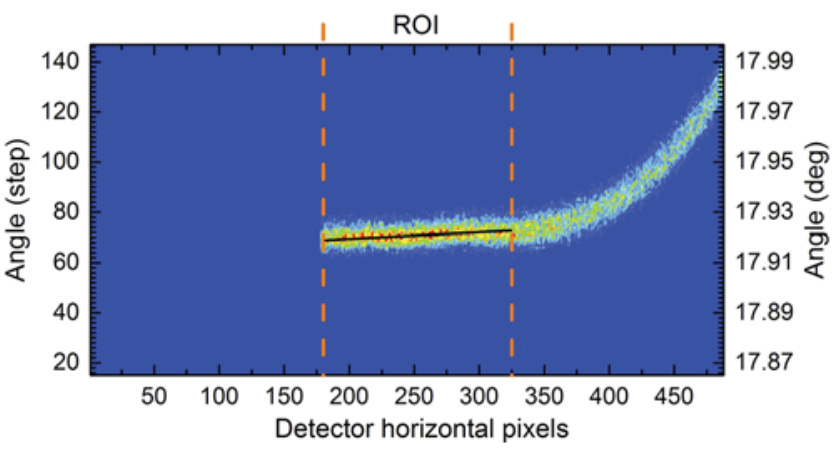

FIG. 13. Two-dimensional image of the Ru $K \alpha_{1}$ X-ray photons measured with the DuMond spectrometer equipped with a $\mathrm{Si}(111)$ crystal. The dashed lines show the region of interest $(\mathrm{ROI})$ with in black the fitted solid line. 
registered. This is caused by the crystal bender mechanics that covers part of the crystal (see Sec. II C). The size of the covered crystal area depends on the Bragg angle and increases with increasing angle. The curved line of the detected photons, visible on the right side of the image, is due to the distortion of the crystal bending toward the side of the crystal, as previously described in the crystal bender section (Sec. II C).

To derive a XES spectrum, only the part of the detector where the image of the X-rays is a straight line is used. This can be achieved by defining a region of interest (ROI) on the $2 \mathrm{D}$-detector matrix. To eliminate a widening of the spectral lines caused by the imperfectly curved parts of the crystal surface (see Sec. II C) which produce tilted $2 \mathrm{D}$-images, the integration of the events along the horizontal axis is made first by fitting a linear or parabolic function to the measured data belonging to the region of interest and then by integrating the fitted function between the lower and upper limits of the ROI. Such a fitted curve is shown in Fig. 13 (black solid line). The range of the ROI is selected in order to obtain the smallest linewidth and the largest possible number of counts. The observed tilt of the photons image is caused by crystal misalignment.

The recorded angular spectra are analyzed by a least-squares fitting procedure, using Voigt functions to fit the measured spectral lines. Voigt functions are employed because they result from the convolution of the Lorentzian profiles, describing the natural X-ray transition line shapes with the Gaussian function representing the instrumental response of the spectrometer. For illustration, the fit

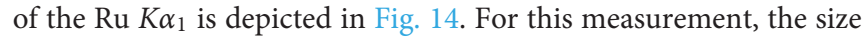
of the synchrotron radiation beam spot at the sample position was estimated using an X-ray eye device to be $100 \times 100 \mu \mathrm{m}^{2}$ in the horizontal and vertical direction, respectively. In the fit, the Lorentzian width of the $K \alpha_{1}$ line was kept fixed at the value $(7.2 \mathrm{eV})$ derived from the atomic level width reported by Campbell and Papp, ${ }^{43}$ while

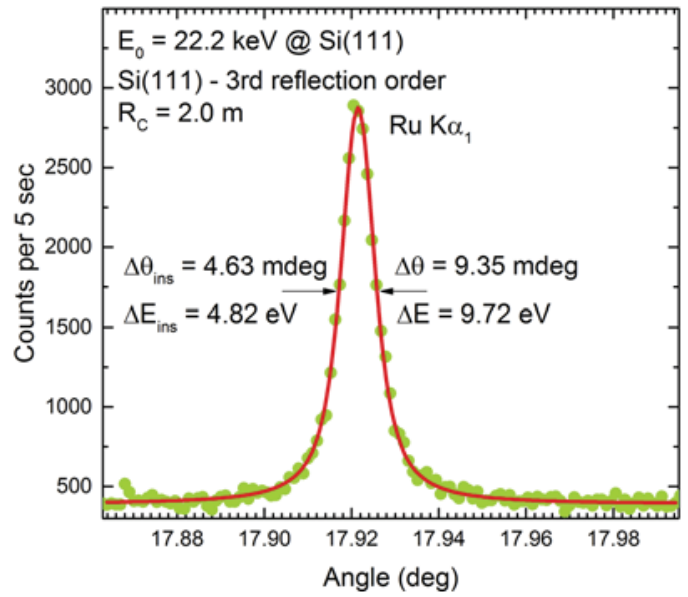

FIG. 14. The Ru K $\alpha_{1} \mathrm{X}$-ray emission line (circle) measured in 3rd order of diffraction with the $\mathrm{Si}(111)$ crystal. The fluorescence from the Ru metal foil was produced by setting the Si(111) channel-cut monochromator to $22.2 \mathrm{keV}$. The circles stand for the experimental data and the solid line for the fit of the Ru $K \alpha$ line with the Voigt function. $\Delta E$ is the total energy resolution resulting from the convolution of the Gaussian instrumental broadening (width $\Delta E_{\text {ins }}$ ) with the Lorentzian natural shape of the transition. the parameters of the linear background, peak intensity, peak position, and instrumental Gaussian width were used as free fitting parameters.

The influence of angular divergence of the X-rays $(\Delta \theta)$ on the instrumental energy resolution can be derived from the Bragg law [Eq. (1)] by calculating the derivative of $E$ as a function of the Bragg angle $\theta_{B}$, which leads to

$$
\Delta E=E \cot \theta_{B} \Delta \theta
$$

Because parameters such as source size, crystal rocking curve profile, crystal size, and its deformation influence effectively on the angular divergence of X-rays, this equation can be interpreted to represent the energy resolution of the crystal spectrometer that work in the DuMond $\theta-2 \theta$ configuration. Due to the scanning of the Bragg angle, the detector pixel size has no direct influence on the energy resolution.

The angular instrumental (Gaussian) contribution to $\Delta \theta$ was found to be $\Delta \theta_{\text {ins }}=4.63 \mathrm{mdeg}=80.8 \mu \mathrm{rad}$. The corresponding instrumental energy resolution obtained from Eq. (2) is $\Delta E_{\text {ins }}=4.82 \mathrm{eV}$. Considering the uncertainties of $\pm 0.33 \mathrm{eV}$ and $\pm 0.15 \mathrm{eV}$ reported by Campbell and $\mathrm{Papp}^{43}$ for $K$ and $L_{3}\left(K \alpha_{1}\right.$ transition) level widths, respectively, an error of $\pm 0.35 \mathrm{eV}$ is found for the instrumental broadening $\Delta E_{\text {ins }}$.

To crosscheck the value of the instrumental energy resolution obtained from the measurement of the $\mathrm{Ru} K \alpha_{1}$ line, the elastic scattering of the monochromatic SR beam by a thin metallic foil was also measured. The same experimental conditions were used. In particular, the beam was tuned to the same energy as the one of the $\mathrm{Ru}$ $K \alpha_{1}$ transition $(19.279 \mathrm{keV})$. The obtained spectrum is presented in Fig. 15.

The relative energy resolution of the SR beam $\left(\Delta E_{\text {intr }} / E_{0}\right)$ for the used channel-cut $\mathrm{Si}(111)$ crystal monochromator is $1.4 \times 10^{-4}$ (taken from Müller et al. ${ }^{44}$ ), which corresponds to $\Delta E_{\text {intr }}=2.7 \mathrm{eV}$

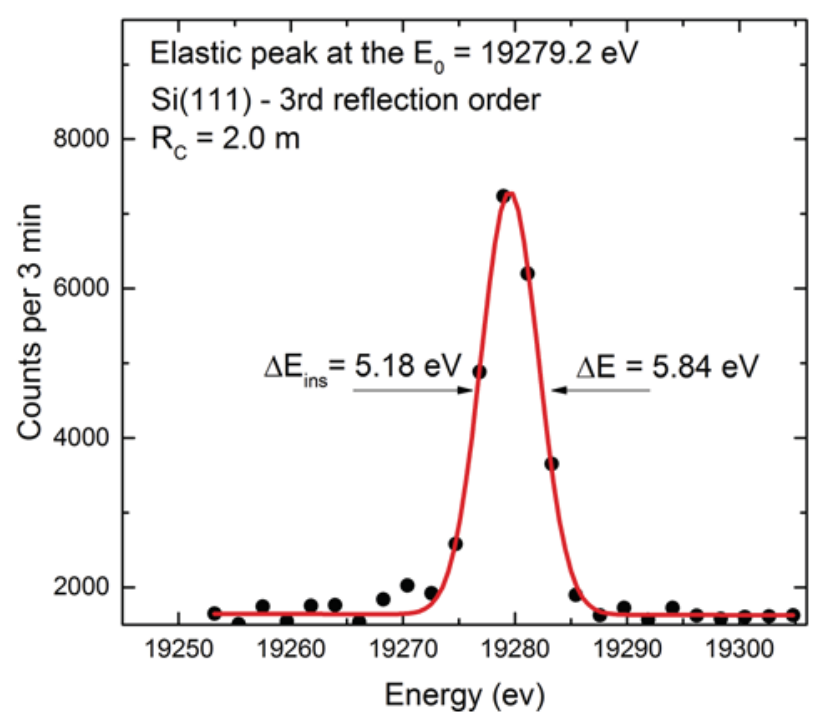

FIG. 15. Elastic scattering peak of the SR beam tuned to the energy of the Ru $K \alpha_{1}$ transition (19.279 keV). The measurement was performed using a $58 \mu \mathrm{m}$ thick $\mathrm{Sn}$ foil as scattering target and a Si(111) crystal in 3rd order of diffraction. 
for the energy $E_{0}=19.279 \mathrm{eV}$. Both the energy distribution of the monochromatic SR beam as well as the instrumental response of the spectrometer can be well reproduced by a Gauss function. Because the function resulting from the convolution of two Gaussians is still a Gauss function whose variance is the sum of the variances of the two convoluted functions, the elastic peak was fitted with a single Gauss function and the energy resolution of the spectrometer was deduced from the relation $\Delta E^{2}=\Delta E_{i n s}^{2}+\Delta E_{\text {intr }}^{2}$. The calibration of the spectrometer for elastic scattering measurement was made with $\mathrm{Ru} K \alpha_{1,2}$ lines. Using the value $\Delta E=5.84 \mathrm{eV}$ provided by the fit, an instrumental broadening $\Delta E_{\text {ins }}=5.18 \mathrm{eV}$ is obtained, which agrees with first method. Comparing the average value of of both methods $\Delta E_{\text {ins }}$ obtained experimentally $(5 \mathrm{eV})$ with the value deduced from the Monte Carlo calculations $(4.73 \mathrm{eV})$, a discrepancy of about $6 \%$ is found. This discrepancy, however, is sufficiently small for enabling the use of the Monte Carlo simulations to guide the further optimization of the spectrometer design.

To optimize the energy resolution, the effect of the focal distance was probed by measuring in 3rd order of diffraction the $\mathrm{Ru}$ $K \alpha_{1}$ X-ray line with the $\mathrm{Si}(111)$ crystal bent to a radius of $2 \mathrm{~m}$, for different distances between the sample position and the crystal. The results of these measurements are presented in Fig. 16, which shows that the best energy resolution is found for a focal distance of about $190.4 \mathrm{~cm}$. This value is in good agreement with the distance between the crystal and the sample position for a crystal bending radius of $2 \mathrm{~m}$. This distance can indeed be calculated from the formula $R_{C} \cos \theta_{B}$ (see Fig. 6) from which a value of $190.29 \mathrm{~cm}$ is found.

The instrumental energy resolution of the DuMond spectrometer was further determined in the range of $16-26 \mathrm{keV}$ by measuring the $K \alpha_{1} \mathrm{X}$-ray spectra of $\mathrm{Zr}, \mathrm{Mo}, \mathrm{Ru}, \mathrm{Pd}, \mathrm{Ag}$, and $\mathrm{Sb}$ metal foils. The measurements were again performed in 3rd order of diffraction with the $\mathrm{Si}(111)$ crystal bent to a radius of $2 \mathrm{~m}$. In the

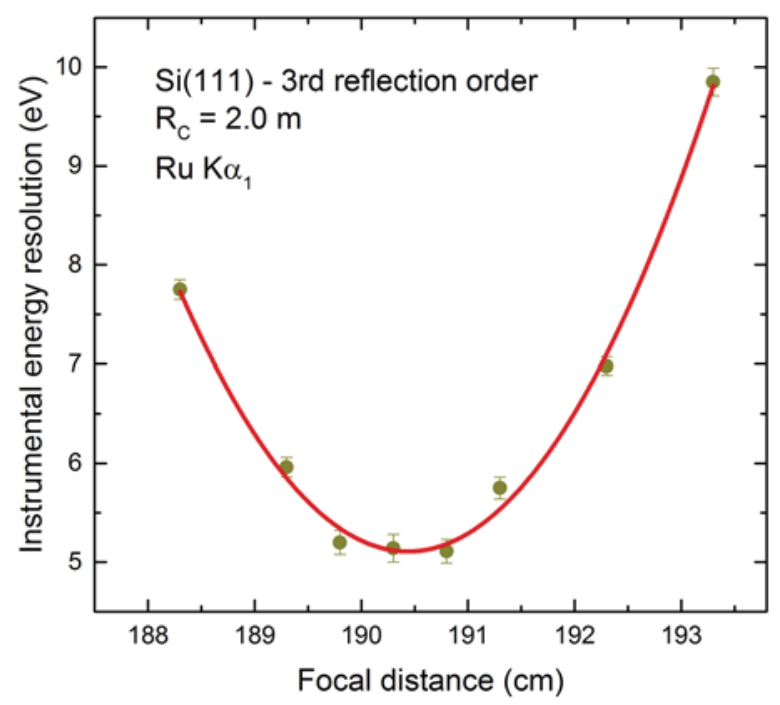

FIG. 16. Variation of the instrumental energy resolution of the DuMond spectrometer as a function of the focal distance. The measurements were performed with the $K \alpha_{1}$ line of $\mathrm{Ru}$ (from a Ru mesh) measured with the $\mathrm{Si}(111)$ crystal $\left(R_{C}=2 \mathrm{~m}\right)$ in 3 rd order of diffraction. The solid line represents the 2 nd order polynomial used to fit the data.

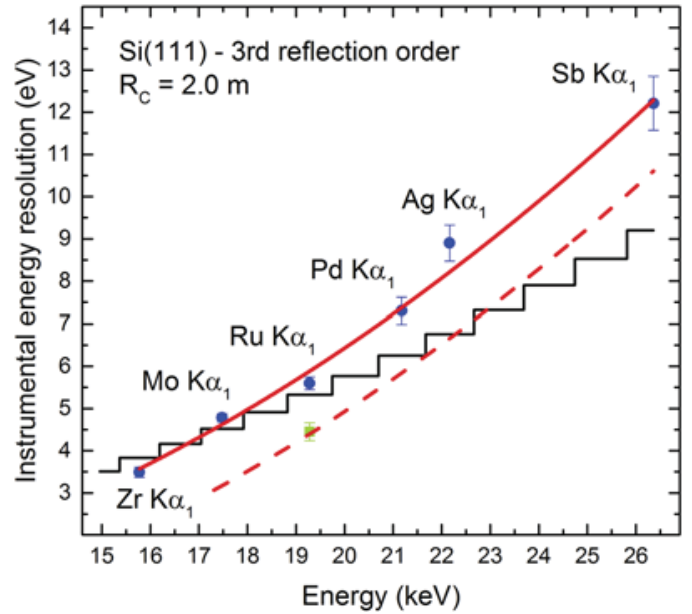

FIG. 17. Variation of the instrumental energy resolution of the DuMond spectrometer as a function of X-ray energy. The measurements were performed with the $\mathrm{Si}(111)$ crystal $\left(R_{C}=2 \mathrm{~m}\right)$ in $3 \mathrm{rd}$ order of diffraction. The solid line represents the 2nd order polynomial used to fit the experimental data. The single square point represents instrumental energy resolution of $\mathrm{Ru} \mathrm{K} \alpha_{1}$ measured for X-ray beam spot on the sample of $100 \times 100 \mu \mathrm{m}^{2}$, and the dotted line is extrapolation of the experimental data fit to that $X$-ray spot size. The black stepped line represents the $K$-shell core-hole lifetime broadening $(\Gamma)$ of elements with $Z=40-50$ reported by Campbell and Papp. ${ }^{43}$

measurements, the beam energy was set above the absorption edge of each studied element. The size of the X-ray beam spot on the sample was $150 \times 150 \mu \mathrm{m}^{2}$. The results are presented in Fig. 17 where the solid line represents the second order polynomial used to fit the data, while the black stepped line is the $K$-shell core-hole lifetime broadening $(\Gamma)$ reported by Campbell and Papp. ${ }^{43}$ As shown, a good agreement is observed between the fit and the data, exhibiting quadratic dependence of the instrumental energy resolution of Laue-type spectrometers. The single square point represents instrumental energy resolution of $\mathrm{Ru} K \alpha_{1}$ measured for X-ray beam spot on the sample of $100 \times 100 \mu \mathrm{m}^{2}$. The dotted line is extrapolation of the experimental data fit to that X-ray spot size. The extrapolation shows that the reached instrumental energy resolution of the DuMond spectrometer is better than the core-hole lifetime broadening in the energy range below $23 \mathrm{keV}$. Additionally, the extrapolated energy resolution of Mo $K \alpha_{1}$ for the DuMond spectrometer (which is $3 \mathrm{eV}$ ) is smaller than value measured using reflection-type spectrometer with $\mathrm{Ge}(999)$ crystal $(3.5-4 \mathrm{eV}){ }^{23}$

Finally, the influence of the crystal radius on the instrumental energy resolution was probed, using the $\mathrm{Ru} K \alpha_{1} \mathrm{X}$-ray line measured in 3rd order of diffraction with the Si(111) crystal for different bending radii. The results of these measurements are presented in Fig. 18. As shown, the energy resolution improves when the crystal radius is increased. A change of the bending radius from 1.5 to $2 \mathrm{~m}$ improves the energy resolution by a factor of 1.4, whereas the peak intensity is reduced simultaneously by a factor of 1.5. A further increase of the crystal radius from 2 to $2.5 \mathrm{~m}$ decreases the peak intensity by a factor 1.5 , while the energy resolution is improved by $5 \%$. Therefore, the best compromise between energy resolution and peak intensity is achieved for a crystal radius equal to $2 \mathrm{~m}$. 


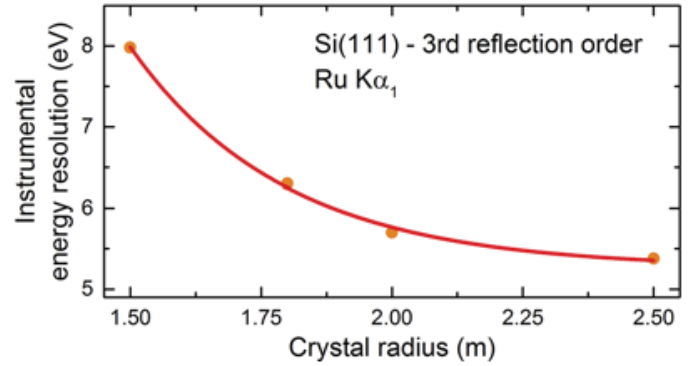

FIG. 18. Variation of the instrumental energy resolution of the DuMond spectrometer as a function of the bending radius of the crystal. The measurements were performed with the Si(111) crystal in 3rd order of diffraction. The solid line represents an exponential fit to the data.

\section{FIRST SPECTROMETER APPLICATION}

As a first application of the novel DuMond-type X-ray spectrometer, the $K \beta_{1,3}$ and $K \beta_{2} \mathrm{X}$-ray emission spectra of metallic $\mathrm{Ru}$ and several $\mathrm{Ru}$ compounds were measured. The spectra were recorded in the 3 rd order of diffraction with a $\mathrm{Si}(111)$ crystal $\left(R_{C}\right.$ $=2 \mathrm{~m}$ ). The monochromatic SR beam energy was tuned to $0.1 \mathrm{keV}$ above the $\mathrm{Ru} K$-edge. The data were collected for 10 s/angular point.

Figure 19 shows the $K \beta_{1,3}$ X-ray spectrum of metallic $\mathrm{Ru}$ (mesh sample). As shown, the two components of the fine-structure doublet (energy separation of $22.1 \mathrm{eV}$ ) are well resolved.

The $K \beta_{2} \mathrm{X}$-ray lines of ruthenium in different oxidation states $\left(\mathrm{Ru}^{0}, \mathrm{Ru}^{\mathrm{IV}} \mathrm{O}_{2}\right.$, and $\left.\mathrm{Ru}^{\mathrm{III}} \mathrm{Cl}_{3}\right)$ were then measured and presented. As shown in Fig. 20, the $K \beta_{2}$ transitions, which are very sensitive to the chemical environment, are slightly shifted in energy depending on the oxidation state of the ruthenium. Note that for $4 \mathrm{~d}$ elements, the $\mathrm{VtC} K \beta_{2} \mathrm{X}$-ray lines are about 25 times weaker than the $K \alpha_{1}$ lines. These measurements demonstrate thus that the sensitivity of the novel spectrometer is high enough to extract the weak VtC transitions of $4 \mathrm{~d}$ elements from the background. In other words, the DuMond spectrometer presented in this paper permits to

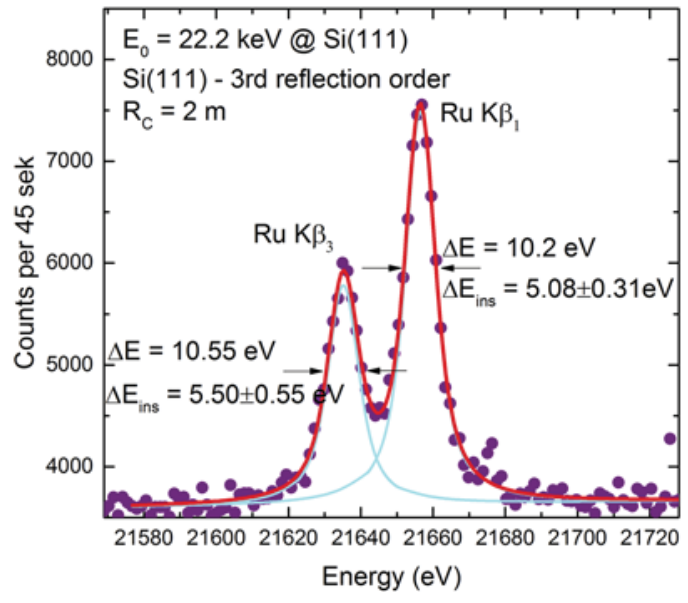

FIG. 19. The Ru $K \beta_{1,3} \mathrm{X}$-ray emission lines measured in 3rd order of diffraction with the Si(111) crystal bent to a radius of $2 \mathrm{~m}$.

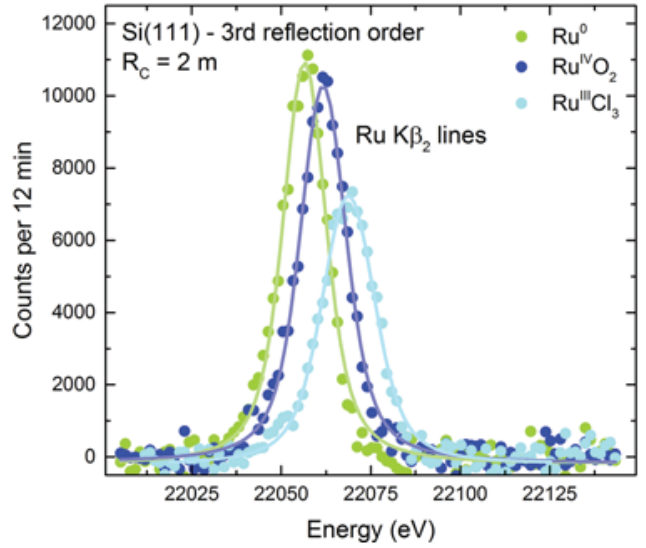

FIG. 20. The Ru $K \beta_{2} X$-ray emission lines of different ruthenium compounds, measured in 3 rd order of diffraction with the $\mathrm{Si}(111)$ crystal bent to radius of $2 \mathrm{~m}$.

investigate the valence electrons of $4 \mathrm{~d}$ transition metals under in situ conditions.

\section{SUMMARY}

In this paper, a high energy resolution Laue-type crystal spectrometer installed at the SuperXAS beamline of the Swiss Light Source was presented. The novel X-ray spectrometer was designed for XES measurements in the 15-26 keV energy range, with one of the main aims being the measurement of the weak VtC transitions of $4 \mathrm{~d}$ elements and compounds. To optimize the design and performance of the novel instrument, numerous simulations based on an X-ray-tracing Monte Carlo code were performed. The predictions of the Monte Carlo simulations were compared with the results of experimental studies concerning mainly the instrumental resolution of the spectrometer. The average value of instrumental energy resolution obtained experimentally is $\Delta E_{\text {ins }}=5 \mathrm{eV}$. The ability of the spectrometer to measure the weak $\mathrm{VtC}$ transitions of $4 \mathrm{~d}$ elements was assessed and validated by successful measurements of the $K \beta_{2} \mathrm{X}$-ray lines of metallic Ru and several Ru compounds in different oxidation states. The measurements show also that the reached energy resolution of the spectrometer allow to observe chemical environment of atomic core.

\section{ACKNOWLEDGMENTS}

J.-Cl.D. and J.H. as well as M.N. would like to acknowledge the financial support of the Swiss National Science Foundation (Grant Nos. 200020-146739 and 200021-159555, respectively). The Swiss Light Source is thanked for provision of beamtime at the SuperXAS beamline.

\section{REFERENCES}

${ }^{1}$ J. A. van Bokhoven, X-ray Absorption and X-ray Emission Spectroscopy (John Wiley \& Sons, 2016).

${ }^{2}$ U. Bergmann and P. Glatzel, Photosynth. Res. 102, 255 (2009).

${ }^{3}$ G. Vanko, P. Glatzel, V.-T. Pham, R. Abela, D. Grolimund, C. N. Borca, S. L. Johnson, C. J. Milne, and C. Bressler, Angew. Chem., Int. Ed. Engl. 49, 5910 (2010). 
${ }^{4}$ K. M. Lancaster, M. Roemelt, P. Ettenhuber, Y. Hu, M. W. Ribbe, F. Neese, U. Bergmann, and S. DeBeer, Science 334, 974 (2011).

${ }^{5}$ P. Glatzel, J. Singh, K. O. Kvashnina, and J. A. van Bokhoven, J. Am. Chem. Soc. 132, 2555 (2010).

${ }^{6}$ J. Szlachetko, D. Banas, A. Kubala-Kukus, M. Pajek, W. Cao, J.-Cl. Dousse, J. Hoszowska, Y. Kayser, M. Szlachetko, M. Kavcic, M. Salome, and J. Susini, J. Appl. Phys. 105, 086101 (2009).

${ }^{7}$ G. Doring, C. Sternemann, A. Kaprolat, A. Mattila, K. Hamalainen, and W. Schulke, Phys. Rev. B 70, 085115 (2004).

${ }^{8}$ V. A. Safonov, L. N. Vykhodtseva, Y. M. Polukarov, O. V. Safonova, G. Smolentsev, M. Sikora, S. G. Eeckhout, and P. Glatzel, J. Phys. Chem. B 110, 23192 (2006).

${ }^{9}$ J. Szlachetko, K. Michalow-Mauke, M. Nachtegaal, and J. Sa, J. Chem. Sci. 126, 511 (2014).

${ }^{10}$ P. Glatzel and U. Bergmann, Coord. Chem. Rev. 249, 65 (2005).

${ }^{11}$ C. J. Doonan, L. Zhang, C. G. Young, S. J. George, A. Deb, U. Bergmann, G. N. George, and S. P. Cramer, Inorg. Chem. 44, 2579 (2005).

${ }^{12}$ I. Lezcano-González, R. Oord, M. Rovezzi, P. Glatzel, S. W. Botchway, B. M. Weckhuysen, and A. M. Beale, Angew. Chem., Int. Ed. 55, 5215 (2016).

${ }^{13}$ B. Ravel, A. J. Kropf, D. Yang, M. Wang, M. Topsakal, D. Lu, M. C. Stennett, and N. C. Hyatt, Phys. Rev. B 97, 125139 (2018).

${ }^{14}$ W. H. Bragg and W. L. Bragg, Proc. R. Soc. London, Ser. A 88, 428 (1913).

${ }^{15}$ H. H. Johann, Z. Phys. 69, 185 (1931).

${ }^{16}$ T. Johansson, Z. Phys. 82, 507 (1933).

${ }^{17}$ L. von Hamos, Ann. Phys. 409, 716 (1933).

${ }^{18}$ L. von Hamos, Ann. Phys. 411, 252 (1934).

${ }^{19}$ Y. Cauchois, J. Phys. Radium 3, 320 (1932).

${ }^{20}$ J. W. M. DuMond, Rev. Sci. Instrum. 18, 626 (1947).

${ }^{21}$ B. L. Henke, E. M. Gullikson, and J. C. Davis, At. Data Nucl. Data Tables 54, 181 (1993).

${ }^{22}$ See http://cxro.lbl.gov for CXRO: The Center for X-ray Optics.

${ }^{23}$ F. A. Lima, R. Bjornsson, T. Weyhermuller, P. Chandrasekaran, P. Glatzel, F. Neese, and S. DeBeer, Phys. Chem. Chem. Phys. 15, 20911 (2013).

${ }^{24}$ M. S. del Río and R. J. Dejus, Proc. SPIE 8141, 814115 (2011).

${ }^{25}$ M. S. del Río, C. Ferrero, and V. Mocella, Proc. SPIE 3151, 312 (1997).

${ }^{26}$ M. S. del Río, Proc. SPIE 3448, 230 (1998).

${ }^{27}$ G. Zschornack, Handbook of X-ray Data (Springer-Verlag Berlin Heidelberg, 2007).

${ }^{28}$ J. Sa, High-Resolution XAS/XES Analyzing Electronic Structures of Catalysts (CRC Press Taylor Francis Group, 2015).

${ }^{29}$ G. L. Borchert, J. Bojowald, A. Ercan, H. Labus, T. Rose, and O. W. B. Schult, Nucl. Instrum. Methods Phys. Res., Sect. A 245, 393 (1986).

${ }^{30}$ B. Perny, J.-Cl. Dousse, M. Gasser, J. Kern, R. Lanners, C. Rheme, and W. Schwitz, Nucl. Instrum. Methods Phys. Res., Sect. A 267, 120 (1988).

${ }^{31}$ C. Herren and J.-Cl. Dousse, Phys. Rev. A 56, 2750 (1997).

${ }^{32}$ T. Ludziejewski, J. Hoszowska, P. Rymuza, and Z. Sujkowski, Nucl. Instrum. Methods Phys. Res., Sect. B 63, 494 (1992).

${ }^{33}$ K. M. Crowe and R. E. Shafer, Rev. Sci. Instrum. 38, 1 (1967).
${ }^{34}$ W. Beer, K. Bos, G. D. Chambrier, K. L. Giovanetti, P. F. A. Goudsmit, B. V. Grigoryev, B. Jeckelmann, L. Knecht, L. N. Kondurova, J. Langhans, H. J. Leisi, P. M. Levchenko, V. I. Marushenko, A. F. Mezentsev, H. Obermeier, A. A. Petrunin, U. Rohrer, A. G. Sergeev, S. G. Skornjakov, A. I. Smirnov, E. Steiner, G. Strassner, V. M. Suvorov, and A. Vacchi, Nucl. Instrum. Methods Phys. Res., Sect. A 238, 365 (1985)

${ }^{35}$ G. L. Borchert, W. Scheck, and O. W. B. Schult, Nucl. Instrum. Methods 124, 107 (1975).

${ }^{36}$ R. Eichler, B. Aas, W. Beer, I. Beltrami, P. Ebersold, T. von Ledebur, H. Leisi, W. Sapp, J.-Cl. Dousse, J. Kern, and W. Schwitz, Phys. Lett. B 76, 231 (1978).

${ }^{37}$ K. Widmann, P. Beiersdorfer, G. V. Brown, J. R. C. L. Urrutia, and V. Decaux, Rev. Sci. Instrum. 68, 1087 (1997).

${ }^{38}$ M. Itou, N. Hiraoka, T. Ohata, M. Mizumaki, A. Deb, Y. Sakurai, and N. Sakai, Nucl. Instrum. Methods Phys. Res., Sect. A 467, 1109 (2001).

${ }^{39}$ N. Hiraoka, M. Itou, T. Ohata, M. Mizumaki, Y. Sakurai, and N. Sakai, J. Synchrotron Radiat. 8, 26 (2001).

${ }^{40}$ N. Hiraoka, T. Buslaps, V. Honkimaki, and P. Suortti, J. Synchrotron Radiat. 12, 670 (2005).

${ }^{41}$ J. F. Seely, J. L. Glover, L. T. Hudson, Y. Ralchenko, A. Henins, N. Pereira, U. Feldman, C. A. D. Stefano, C. C. Kuranz, R. P. Drake, H. Chen, G. J. Williams, and J. Park, Rev. Sci. Instrum. 85, 11 D618 (2014).

${ }^{42}$ M. Szlachetko, M. Berset, J.-Cl. Dousse, J. Hoszowska, and J. Szlachetko, Rev. Sci. Instrum. 84, 093104 (2013).

${ }^{43}$ J. L. Campbell and T. Papp, At. Data Nucl. Data Tables 77, 1 (2001).

${ }^{44}$ O. Müller, M. Nachtegaal, J. Just, D. Lutzenkirchen-Hecht, and R. Frahm, J. Synchrotron Radiat. 23, 260 (2016).

${ }^{45}$ P. M. Abdala, O. V. Safonowa, G. Wiker, W. van Beek, H. Emerich, J. A. van Bokhoven, J. Sa, J. Szlachetko, and M. Nachtegaal, Chimia 66, 699 (2012).

${ }^{46}$ E. Kleymenov, J. A. van Bokhoven, C. David, P. Glatzel, M. Janousch, R. Alonso-Mori, M. Studer, M. Willimann, A. Bergamaschi, B. Henrich, and M. Nachtegaal, Rev. Sci. Instrum. 82, 065107 (2011).

${ }^{47}$ J. Szlachetko, M. Nachtegaal, E. de Boni, M. Willimann, O. Safonova, J. Sa, G. Smolentsev, M. Szlachetko, J. A. van Bokhoven, J.-Cl. Dousse, J. Hoszowska, Y. Kayser, P. Jagodziński, A. Bergamaschi, B. Schmitt, C. David, and A. Lücke, Rev. Sci. Instrum. 83, 103105 (2012).

${ }^{48}$ T. Taguchi, C. Brönnimann, and E. F. Eikenberry, Powder Diffr. 23, 101 (2008).

${ }^{49}$ M. Krisch, A. Freund, G. Marot, and L. Zhang, Nucl. Instrum. Methods Phys. Res., Sect. A 305, 208 (1991).

${ }^{50}$ P. Jagodziński, M. Pajek, D. Banaś, H. Beyer, M. Trassinelli, and T. Stohlker, Nucl. Instrum. Methods Phys. Res., Sect. A 753, 121 (2014).

${ }^{51}$ B. E. Warren, X-ray Diffraction (Dover Publications, Inc., New York, 1969).

${ }^{52}$ W. H. Zachariasen, Theory of X-ray Diffraction in Crystals (Dover Publications, New York, 1967).

${ }^{53}$ M. Matsumoto and T. Nishimura, ACM Trans. Model. Comput. Simul. 8, 3 (1998).

${ }^{54}$ A. H. Compton and S. K. Allison, X-rays in Theory and Experiment (Van Nostrand, New York, 1935).

${ }^{55}$ O. Schult, Z. Phys. 158, 444 (1960). 